\title{
A welcome address for the new criteria
}

\section{Dirkjan van Schaardenburg, ${ }^{1}$ Ben A C Dijkmans ${ }^{2}$}

After lively presentations at the European League Against Rheumatism (EULAR) and American College of Rheumatology (ACR) meetings, the new criteria for rheumatoid arthritis (RA) now appear in print accompanied by methodological reports. ${ }^{12}$ The interested reader can thereby follow the entire process from beginning to end. It has been a huge task and the members of the ACR/EULAR joint taskforce deserve our warm congratulations for the result, which is easy to absorb because the reasoning is clear and the manuscripts are well written. Through the combination of a first phase with a data-driven approach followed by a second phase using new consensus methodology, we can be confident that a high-quality product has been delivered.

The main question is why the 1987 ACR criteria for the classification of RA should be revised. The 1987 criteria were derived from patients with RA with mostly longstanding disease and identified features that best discriminated these patients from those with a variety of other rheumatic disorders. In the meantime, the focus of the management of RA has shifted from alleviating the consequences of joint damage to early diagnosis and intervention. The old criteria were increasingly viewed as not helpful in achieving the new goal. The aim of the endeavour of experts leading to new classification criteria was to define the group of patients with a high risk of developing persistent and erosive disease who will likely benefit from the rapid initiation of antirheumatic treatment.

\section{CONSEQUENCES FOR TREATMENT}

What these criteria actually attempt to do is to separate, at an early stage, patients

\footnotetext{
${ }^{1}$ Department of Rheumatology, Jan van Breemen Institute, Amsterdam, The Netherlands ${ }^{2}$ Department of Rheumatology, VU Free University Medical Center, Amsterdam, The Netherlands

Correspondence to Dirkjan van Schaardenburg, Department of Rheumatology, Jan van Breemen Institute, Jan van Breemenstraat 2, Amsterdam 1056 $A B$, The Netherlands;

d.v.schaardenburg@janvanbreemen.nl
}

with undifferentiated arthritis into two groups-one with a poor prognosis and one with a good prognosis. The underlying message could be to treat those with a poor prognosis (RA) aggressively and those with a good prognosis (not RA) symptomatically. Rheumatologists have already become used to the successful treatment of active early RA with combination therapies, sometimes including biological agents. ${ }^{3-5}$ With the advent of new criteria, the door is now open to try aggressive therapy in patients with less active disease. However, we may encounter some obstacles to success. Adverse events such as infections due to immune suppression will be less acceptable in patients with only a few involved joints, while alternative treatment options are available. Also, these patients will generally be more reluctant to accept intensive treatment regimens than patients with severe disease. Moreover, it will be more difficult to demonstrate efficacy of a given therapy in patients entering a study with low disease activity. Numerous patients who just fulfil the new criteria will, at the same time, fulfil existing remission criteria. Accordingly, we will have to define suitable treatment goals and remission criteria. ${ }^{6}$

Conversely, lack of fulfilment of the criteria could lead to a more conservative treatment policy in patients who do not cross the line of the required 6 -point score. This does not need to be a problem since patients can fulfil the criteria at any later point in time should their disease become more active. How far patients with arthritis who do not fulfil the criteria over a longer period and do not receive treatment are at risk of developing erosions needs to be studied.

Some patients will be treated with a disease-modifying antirheumatic drug (DMARD) anyway, even if they do not fulfil the criteria, since clinical practice is often ahead of criteria and guidelines. Consider a new patient presenting with three painful and swollen metacarpophalangeal, proximal interphalangeal or metatarsophalangeal joints for more than 6 weeks, C reactive protein (CRP) in the normal range, with normal x-rays and a low positive anticitrullinated peptide antibody (ACPA) who does not have RA according to the new tree algorithm. A brief round among rheumatologists in our departments showed that 13 out of 16 would start methotrexate in such a patient, which means that the gold standard of methotrexate initiation that was used as 'proof' for the presence of RA is not a static feature. Looking back at the period 1995-2000, rheumatologists from our group tended to treat patients with undifferentiated arthritis a lot less aggressively than patients with RA as defined by the old ACR criteria. ${ }^{7}$

The abovementioned example illustrates that the diagnosis and treatment of the individual patient depends more on the clinical perception of the rheumatologist than on classification according to criteria. Naturally, physicians can decide to follow the idea behind the criteria, prescribing methotrexate to all patients who fulfil the criteria and withholding methotrexate from those who do not. If the criteria are indeed capable of optimally distinguishing patients with good and bad prognosis, this strategy will lead to good results without overtreatment.

\section{OTHER CONSEQUENCES OF CHANGE}

How will the introduction of the new criteria affect the interpretation of existing trial results, guidelines, epidemiological studies and patient benefits that were based on the application of the old ACR criteria for RA? ${ }^{8}$ The expectation is that the new criteria will be more sensitive, especially in early disease, leading to more patients with RA with mild disease. We therefore do not expect that patients who have participated in past trials would not fulfil the new criteria. However, the extent to which past RA trial results can be generalised to all patients with RA, defined by old or by new criteria, depends on how much these other patients resemble the trial participants. Most trials in RA have been conducted in patients with very active disease, often with a requirement of at least six or eight swollen joints. Thus, the optimal management of mildly active RA is not yet known.

Future versions of guidelines of societies for rheumatology may state, with the new criteria in mind, that all new patients with RA should be treated with at least methotrexate. While this reflects current practice in most cases, rheumatologists may risk facing complaints if they choose to treat less aggressively. 
Future epidemiological studies of RA may suffer from lack of comparability with older studies. However, studies of the incidence of RA that measured the separate items of the criteria sets in the past, such as the British NOAR register, ${ }^{9}$ can continue to compare the disease phenotype. The measurement of prevalence in cross-sectional surveys of longstanding disease will also not be affected greatly since the new criteria accommodate the inclusion of patients with past evidence of RA, whether presently active or quiescent and with or without joint damage. In this respect, the choice to let the presence of typical RA erosions suffice to classify a patient as RA according to the new criteria was a good decision.

Additional validation studies will now be performed. Such studies will show whether the new criteria perform as well in other cohorts as in the three cohorts that have been used for that purpose, and how they compare with the old criteria. However, even if they do not perform better than the old criteria, the new criteria still have the major advantage that they measure disease characteristics that are relevant to early disease.

Many clinical epidemiological studies of undifferentiated arthritis or RA are currently being performed that were designed with the old ACR criteria as the standard. The analysis and reporting of such studies may be complicated should the editors of rheumatology journals demand that, from now on, all studies on RA are to report with the new criteria. Although the use of the new criteria are to be promoted as much as possible, a transition period should allow studies to report using the old criteria if they have already started their inclusion of patients at the time of the publication of the criteria.

There is also a possible societal impact of the introduction of the new criteria to consider. The trend to give biological agents to a growing portion of new patients with RA will probably increase through this introduction. The direct effect of more expensive medications on the cost to society is obvious, whereas the suggested net savings of the total costs of the RA 'patient career' by early-and possibly temporary-treatment with biological agents remains to be demonstrated. In some instances, patient benefits such as easier access to certain facilities or reimbursements will be easier to obtain in cases where these are dependent on the diagnosis of RA.

\section{WHAT IS REALLY NEW?}

The fundamentally different approach to the classification of RA, as was made quite clear, is the novel practical focus on recognising early disease with a poor prognosis. When we look at the separate new criteria, the most conspicuous newcomer among them is ACPA, which adds for the first time a pathogenetic element to the criteria. ${ }^{10}{ }^{11} \mathrm{ACPA}$ proved to have the strongest relation of all the examined biomarkers to the gold standard of methotrexate use, and was shown before to be among the most powerful of prognostic factors in RA. ${ }^{12}$ Since ACPA is also related to most of the known genetic factors associated with RA, ACPA-positive and ACPA-negative RA appear to be the major subsets of the disease, both from the viewpoints of pathogenesis and of severity. ${ }^{10}$

Following this line, the next step in the future development of RA criteria should be to further define the subsets of RA according to combinations of clinical expressions and new biomarkers with therapeutic or prognostic relevance..$^{13}$ Certain therapies may prove to be beneficial in specific subsets, which would be a welcome refinement to the present 'one bullet for all' strategy.

The imaging of inflammation in RA by sonography, MRI, positron emission tomography scanning or other modalities may be able to add some sensitivity to the physical examination concerning the sites and extent of inflammation. ${ }^{14}$ Overall, the main progress in the next years should come from new and specific biomarkers of the RA inflammatory process itself, which is still largely not understood.

In the meantime, the application of the new criteria would be greatly helped if ACPA testing became standardised and less costly. Several types of ACPA appear to have a similar diagnostic and prognostic value ${ }^{15}$ but most of them are not commercially available. The cost of the commercially available tests is prohibitive to widespread use in many countries. Reasonably standardised ELISAs for IgM rheumatoid factor are widely available at low cost, and that is what we need for the more sensitive and specific ACPA tests.

\section{METHODOLOGICAL MATTERS}

The authors selected the prescription of methotrexate as the gold standard for RA, but what if they had additionally allowed the prescription of other DMARDs to qualify as RA? One might expect that drugs such as sulfasalazine or antimalarial agents are given to patients with milder disease than is present in those who receive methotrexate. The addition of sulfasalazine and antimalarial drugs to the gold standard would therefore have rendered the criteria more sensitive. Alternatively, the choice of sulfasalazine might have led to the inclusion of more patients who actually had psoriatic arthritis or spondyloarthropathy, and the choice of antimalarial drugs to the inclusion of more patients who actually had systemic lupus erythematosus, thus reducing specificity.

The authors state that, if another diagnosis such as psoriatic arthritis better fits the patient, the criteria should not be applied. However, how should we classify the patient with psoriasis and arthritis not in the distal interphalangeal joints but only in more proximal hand joints? Should the decision depend on the severity of the skin psoriasis or possibly on imaging evidence of involvement of the entheses? ${ }^{16}$

It is noteworthy that symmetry of joint involvement had no added value in the analysis of phase I, leading to the deletion of this member of the old set of criteria. Intuitively, symmetry may be deemed important because the opposite-asymmetry-is often a feature of spondyloarthropathy, a condition which needs to be distinguished from RA. Spondyloarthropathy is more prevalent at northern latitudes, which may explain in part the large differences between the composition of the various cohorts that were used to derive the criteria. In the cohort from Norway, for example, we see only $14 \%$ prevalence of ACPA. Given the fact that eight out of nine cohorts in the study come from Europe (and three even from one country), it is remarkable that such large differences are seen between the cohorts concerning the features of their patients with early arthritis. The question arises whether the result would have been different if other cohorts in other countries had been used for the development of the criteria. However, although other cohorts would have yet another patient mix, in the end the borderline between what is defined as RA or non-RA would have to come very close to the present result owing to the second phase of expert consensus. A potentially more troublesome difference between the cohorts could be a different inclination to prescribe methotrexate, with consequences for the validity of the gold standard. This 
inclination could be assessed by doing surveys of treatment choices for certain patient profiles among the rheumatologists of participating centres.

The instruction in the criteria application to use local reference values for acute phase reactants is unfortunate as it leaves much room for local interpretations of what is normal. Whereas one single reference value should have been set for CRP, erythrocyte sedimentation rate is higher on average in women and at higher ages. ${ }^{17}$ This can easily be accounted for, and a direction here would promote a more uniform application of the criteria.

\section{THE NEXT CRITERIA SET}

In our opinion, the process of developing criteria reflects a lack of insight into the pathogenesis of inflammatory diseases. A comparison can be made by making a set of criteria to diagnose HIV infection or pneumococcal disease without insight into the pathogenesis or the presence of the corresponding microorganism. As soon as we have sufficient insight into the pathogenesis of RA, no further consensus exercises by expert panels will be needed to define RA. Before we reach that stage, our understanding and treatment of RA will evolve in small steps as before. The evaluation of new technologies takes time, so it cannot be foreseen when enough progress will have been made to justify a new version. We can speculate, however, that the next version will not be criteria of RA in general, but rather of a number of subsets of chronic arthritis that will guide the counselling of patients and the making of therapeutic decisions with, hopefully, a lot more precision and success than is possible today.

Provenance and peer review Commissioned; externally peer reviewed.

Competing interests None.

Accepted 6 June 2010

Ann Rheum Dis 2010;69:1577-1579.

doi:10.1136/ard.2010.135335

\section{REFERENCES}

1. Funovits J, Aletaha D, Bykerk V, et al. The 2010 American College of Rheumatology/European League Against Rheumatism classification criteria for rheumatoid arthritis: Methodological Report Phase I. Ann Rheum Dis 2010;69:1589-95.

2. Aletaha D, Neogi T, Silman A, et al. 2010 Rhuematoid arthritis classification criteria: an American College of Rheumatology/European League Against Rheumatism collaborative initiative. Arthritis Rheum 2010;62:2569-81.

3. Boers M, Verhoeven AC, Markusse HM, et al. Randomised comparison of combined step-down prednisolone, methotrexate and sulphasalazine with sulphasalazine alone in early rheumatoid arthritis. Lancet 1997;350:309-18.

4. Goekoop-Ruiterman YP, de Vries-Bouwstra JK, Allaart CF, et al. Comparison of treatment strategies in early rheumatoid arthritis: a randomized trial. Ann Intern Med 2007;146:406-15.

5. Möttönen T, Hannonen P, Leirisalo-Repo M, et al. Comparison of combination therapy with single-drug therapy in early rheumatoid arthritis: a randomised trial. FIN-RACo trial group. Lancet 1999;353:1568-73.

6. van Tuyl LH, Vlad SC, Felson DT, et al. Defining remission in rheumatoid arthritis: results of an initial American College of Rheumatology/ European League Against Rheumatism consensus conference. Arthritis Rheum 2009;61:704-10.

7. Jansen LM, van Schaardenburg $D$, van der Horst-Bruinsma IE, et al. One year outcome of undifferentiated polyarthritis. Ann Rheum Dis 2002; 61:700-3.

8. Arnett FC, Edworthy SM, Bloch DA, et al. The American Rheumatism Association 1987 revised criteria for the classification of rheumatoid arthritis. Arthritis Rheum 1988;31:315-24.

9. Harrison BJ, Symmons DP, Barrett EM, et al. The performance of the 1987 ARA classification criteria for rheumatoid arthritis in a population based cohort of patients with early inflammatory polyarthritis. American Rheumatism Association. $J$ Rheumatol 1998;25:2324-30.

10. Klareskog L, Rönnelid J, Lundberg K, et al. Immunity to citrullinated proteins in rheumatoid arthritis. Annu Rev Immunol 2008;26:651-75.

11. Sebbag M, Chapuy-Regaud S, Auger I, et al. Clinical and pathophysiological significance of the autoimmune response to citrullinated proteins in rheumatoid arthritis. Joint Bone Spine 2004;71:493-502.

12. De Rycke L, Peene I, Hoffman IE, et al. Rheumatoid factor and anticitrullinated protein antibodies in rheumatoid arthritis: diagnostic value, associations with radiological progression rate, and extra-articular manifestations. Ann Rheum Dis 2004;63:1587-93

13. van Baarsen LG, Bos WH, Rustenburg F, et al. Gene expression profiling in autoantibody-positive patients with arthralgia predicts development of arthritis. Arthritis Rheum 2010;62:694-704.

14. Østergaard M, Pedersen SJ, Døhn UM. Imaging in rheumatoid arthritis-status and recent advances for magnetic resonance imaging, ultrasonography, computed tomography and conventional radiography. Best Pract Res Clin Rheumatol 2008;22:1019-44.

15. Ursum J, Nielen MM, van Schaardenburg D, et al. Antibodies to mutated citrullinated vimentin and disease activity score in early arthritis: a cohort study. Arthritis Res Ther 2008;10:R12.

16. Marzo-Ortega H, Tanner SF, Rhodes LA, et al. Magnetic resonance imaging in the assessment of metacarpophalangeal joint disease in early psoriatic and rheumatoid arthritis. Scand J Rheumatol 2009;38:79-83.

17. Miller A, Green M, Robinson D. Simple rule for calculating normal erythrocyte sedimentation rate. BMJ (Clin Res Ed) 1983;286:266. 